\title{
The effect of projections on fractal sets and measures in Banach spaces
}

\author{
WILLIAM OTT $\dagger$, BRIAN HUNT $\ddagger$ and VADIM KALOSHIN $\S$ \\ $\dagger$ Courant Institute of Mathematical Sciences, New York, NY 10012, USA \\ (e-mail: ott@cims.nyu.edu) \\ $\ddagger$ Institute for Physical Science and Technology, University of Maryland, \\ College Park, MD 20742, USA \\ (e-mail: bhunt@ipst.umd.edu) \\ $\S$ Department of Mathematics, California Institute of Technology, Pasadena, \\ CA 91125, USA \\ (e-mail:kaloshin@its.caltech.edu)
}

(Received 19 December 2003 and accepted in revised form 18 June 2005)

\begin{abstract}
We study the extent to which the Hausdorff dimension of a compact subset of an infinite-dimensional Banach space is affected by a typical mapping into a finitedimensional space. It is possible that the dimension drops under all such mappings, but the amount by which it typically drops is controlled by the 'thickness exponent' of the set, which was defined by Hunt and Kaloshin (Nonlinearity 12 (1999), 1263-1275). More precisely, let $X$ be a compact subset of a Banach space $B$ with thickness exponent $\tau$ and Hausdorff dimension $d$. Let $M$ be any subspace of the (locally) Lipschitz functions from $B$ to $\mathbb{R}^{m}$ that contains the space of bounded linear functions. We prove that for almost every (in the sense of prevalence) function $f \in M$, the Hausdorff dimension of $f(X)$ is at least $\min \{m, d /(1+\tau)\}$. We also prove an analogous result for a certain part of the dimension spectra of Borel probability measures supported on $X$. The factor $1 /(1+\tau)$ can be improved to $1 /(1+\tau / 2)$ if $B$ is a Hilbert space. Since dimension cannot increase under a (locally) Lipschitz function, these theorems become dimension preservation results when $\tau=0$. We conjecture that many of the attractors associated with the evolution equations of mathematical physics have thickness exponent zero. We also discuss the sharpness of our results in the case $\tau>0$.
\end{abstract}

\section{Introduction}

Many infinite-dimensional dynamical systems have been shown to have compact finitedimensional attractors $[3,4,26,28,29]$. Such attractors exist for a variety of the evolution equations of mathematical physics, including the Navier-Stokes system, various classes of 
reaction-diffusion systems, nonlinear dissipative wave equations, and complex GinzburgLandau equations. When an attractor is measured experimentally, one observes a 'projection' of the attractor into finite-dimensional Euclidean space. (By projection, we mean simply a mapping to a lower-dimensional space; this mapping may be nonlinear.) This technique of observation via projection leads to a natural and fundamental question. How accurately does the image of the attractor reflect the attractor itself? We address this question from a dimension-theoretic perspective and we consider the following problem. For an attractor of an infinite-dimensional dynamical system, how is its dimension affected by a typical projection into a finite-dimensional Euclidean space?

One may define the dimension of an attractor in many different ways. Setting aside dynamics, the attractor may be viewed as a compact set of points in a metric space. Viewing the attractor in this light, the dimension of the attractor may be defined as the boxcounting dimension or the Hausdorff dimension of the attracting set. Measure-dependent notions of attractor dimension take into account the distribution of points induced by the dynamics and are thought to be more accurately measured from numerical or experimental data. One often analyzes the 'natural measure', the probability measure induced by the statistics of a typical trajectory that approaches the attractor. A natural measure is not known to exist for arbitrary systems, but it does exist for Axiom A attractors and for certain classes of systems satisfying conditions weaker than uniform hyperbolicity. See $[\mathbf{1 4}, \mathbf{3 0}]$ for expository discussions of systems that are known to have natural measures.

The dimension spectrum $\left(D_{q}\right.$ spectrum) characterizes the multifractal structure of an attractor. Given a Borel measure $\mu$ with compact support $X$ in some metric space, for $q \geqslant 0$ and $q \neq 1$ let

$$
D_{q}(\mu)=\lim _{\epsilon \rightarrow 0} \frac{\log \int_{X}[\mu(B(x, \epsilon))]^{q-1} d \mu(x)}{(q-1) \log \epsilon},
$$

provided the limit exists, where $B(x, \epsilon)$ is the ball of radius $\epsilon$ centered at $x$. (If the limit does not exist, define $D_{q}^{+}(\mu)$ and $D_{q}^{-}(\mu)$ to be the lim sup and lim inf, respectively.) Let

$$
D_{1}(\mu)=\lim _{q \rightarrow 1} D_{q}(\mu),
$$

again provided the limit exists. This spectrum includes the box-counting dimension $\left(D_{0}\right)$, the information dimension $\left(D_{1}\right)$, and the correlation dimension $\left(D_{2}\right)$. In particular, when $q=0$ the dimension depends only on the support $X$ of $\mu$ and we write $D_{0}(X)=D_{0}(\mu)$. See $\S 2$ for a discussion of this definition and its relationship to other definitions of $D_{q}$ in the literature.

The goal of this paper is to extend the following theorems, as much as possible, to infinite-dimensional Banach spaces. In all of the results in this paper, 'almost every' is in the sense of prevalence, a generalization of 'Lebesgue almost every' to infinitedimensional spaces. See $\S 2$ and $[\mathbf{1 5}, \mathbf{1 6}]$ for details.

THEOREM 1.1. [27] Let $X \subset \mathbb{R}^{n}$ be a compact set. For almost every function $f \in$ $C^{1}\left(\mathbb{R}^{n}, \mathbb{R}^{m}\right)$, one has

$$
\operatorname{dim}_{H}(f(X))=\min \left\{m, \operatorname{dim}_{H}(X)\right\}
$$

where $\operatorname{dim}_{\mathrm{H}}(\cdot)$ is the Hausdorff dimension. 
THEOREM 1.2. [12] Let $\mu$ be a Borel probability measure on $\mathbb{R}^{n}$ with compact support and let $q$ satisfy $1<q \leqslant 2$. Assume that $D_{q}(\mu)$ exists. Then for almost every function $f \in C^{1}\left(\mathbb{R}^{n}, \mathbb{R}^{m}\right), D_{q}(f(\mu))$ exists and is given by

$$
D_{q}(f(\mu))=\min \left\{m, D_{q}(\mu)\right\} .
$$

For each result, the space $C^{1}\left(\mathbb{R}^{n}, \mathbb{R}^{m}\right)$ can be replaced by any space that contains the linear functions from $\mathbb{R}^{n}$ to $\mathbb{R}^{m}$ and is contained in the (locally) Lipschitz functions. By locally Lipschitz, we mean that the function has a finite Lipschitz constant on each bounded subset of $\mathbb{R}^{n}$; this condition ensures that the dimensions we have discussed do not increase under $f$. Theorem 1.1 extends to nonlinear functions a result of Mattila [21] (generalizing earlier results of Marstrand [20] and Kaufman [19]) that makes the same conclusion for almost every linear function from $\mathbb{R}^{n}$ into $\mathbb{R}^{m}$, in the sense of Lebesgue measure on the space of $m$-by- $n$ matrices. Strictly speaking, Marstrand, Kaufman, and Mattila considered orthogonal projections, but the analogous results for general linear projections follow immediately. Sauer and Yorke [27] prove Theorem 1.2 for the correlation dimension $\left(D_{2}\right)$ and recover (1.1) by invoking a variational principle for Hausdorff dimension [5]. Theorem 1.1 and its predecessors follow from a potentialtheoretic characterization of the dimensions involved. Roughly speaking, the dimension is the largest exponent for which a certain singular integral converges. Theorem 1.2 follows from a similar characterization of $D_{q}$ for $q>1$ [12]; see Definition 2.3.

The potential-theoretic approach only leads to a dimension preservation result for $D_{q}$ if $1<q \leqslant 2$. For $0 \leqslant q<1$ and $q>2$, [12] gives examples for which $D_{q}$ is not preserved by any linear transformation into $\mathbb{R}^{m}$. For $0 \leqslant q<1$, the construction is based on a class of examples constructed independently by Falconer and Howroyd [6] and Sauer and Yorke [27] for which the box-counting dimension is not preserved by any $C^{1}$ function. (An earlier example for which box-counting dimension is not preserved under projection was given by Järvenpää [17].)

When the ambient space is not finite-dimensional, one does not expect a dimension preservation result analogous to Theorem 1.1 or Theorem 1.2 to hold. We use the thickness exponent [13] to study the extent to which the dimension spectrum is affected by projection from a Banach space to $\mathbb{R}^{m}$. This exponent, defined precisely in $\S 2$ and denoted $\tau(X)$, measures how well a compact subset $X$ of a Banach space $B$ can be approximated by finite-dimensional subspaces of $B$, with smaller values of the thickness exponent indicating better approximability. In general one has $\tau(X) \leqslant D_{0}^{+}(X)$, the upper box-counting dimension of $X$, and equality is possible. We expect that the thickness exponent can be shown to be significantly smaller than the box-counting dimension for many attractors of infinite-dimensional systems. Studying the Hölder regularity of embeddings of infinitedimensional fractal sets into finite-dimensional spaces, [13] establishes a bound on the amount the dimension may drop for a typical projection.

THEOREM 1.3. [13] Let $B$ denote a Banach space. Let $X \subset B$ be a compact set with box-counting dimension $d$ and thickness exponent $\tau(X)$. Let $m>2 d$ be an integer, and let $\alpha$ be a real number with

$$
0<\alpha<\frac{m-2 d}{m(1+\tau(X))}
$$


Then for almost every (in the sense of prevalence) bounded linear function (or $C^{1}$ function, or Lipschitz function) $f: B \rightarrow \mathbb{R}^{m}$, there exists $C>0$ such that for all $x, y \in X$,

$$
C|f(x)-f(y)|^{\alpha} \geqslant|x-y| .
$$

For such a function $f$, one has

$$
\frac{m-2 d}{m(1+\tau(X))} \operatorname{dim}(X) \leqslant \operatorname{dim}(f(X)) \leqslant \operatorname{dim}(X)
$$

where $\operatorname{dim}(X)$ represents either the box-counting dimension or Hausdorff dimension.

This theorem generalizes earlier results in [2] and [7].

In Theorem 1.3, the factor by which the dimension may drop is the product of two terms, $(m-2 d) / m$ and $1 /(1+\tau(X))$. The first term depends on the embedding dimension $m$ and converges to one as $m \rightarrow \infty$ while the second term depends intrinsically on $X$ via its thickness exponent. We prove that the Hausdorff dimension is preserved by a typical projection up to a factor of $1 /(1+\tau(X))$. In particular, the factor $(m-2 d) / m$ has been removed. We now state the main theorem for compact subsets of Banach spaces. Because of the possibility of dimension drop, the existence of $D_{q}(\mu)$ does not imply the existence of $D_{q}(f(\mu))$ for functions $f$ satisfying the conclusion of the theorem. We therefore formulate the result in terms of the lower dimension $D_{q}^{-}$.

BANACH Space TheOREM. Let $B$ be a Banach space, and let $M$ be any subspace of the (locally) Lipschitz functions from $B$ to $\mathbb{R}^{m}$ that contains the space of bounded linear functions. Let $X \subset B$ be a compact set with thickness exponent $\tau(X)$. Let $\mu$ be a Borel probability measure supported on X. For almost every (in the sense of prevalence $\dagger$ ) $f \in M$, one has

$$
\operatorname{dim}_{H}(f(X)) \geqslant \min \left\{m, \frac{\operatorname{dim}_{H}(X)}{1+\tau(X)}\right\},
$$

and, for $1<q \leqslant 2$,

$$
D_{q}^{-}(f(\mu)) \geqslant \min \left\{m, \frac{D_{q}^{-}(\mu)}{1+\tau(X)}\right\} .
$$

It is not hard to show that if $f$ is Lipschitz on $X$, then the dimension of $f(X)$ (respectively $f(\mu)$ ) is not greater than the dimension of $X$ (respectively $\mu$ ) for all of the notions of dimension we have discussed. Thus, for sets with thickness exponent zero, the Banach space theorem is a dimension preservation result. Every set $X \subset \mathbb{R}^{n}$ has thickness exponent zero. Thus, the Banach space theorem generalizes Theorems 1.1 and 1.2. Furthermore, it strengthens Theorem 1.2, because, for a prevalent set of functions, (1.3) holds simultaneously for all $1<q \leqslant 2$. On the other hand, suppose $\tau(X)>0$. The Hausdorff dimension of $X$ may be non-computable in the sense that $\operatorname{dim}_{H}(f(X))<\operatorname{dim}_{H}(X)$ for every positive integer $m$ and every sufficiently smooth function $f$. In other words, the Hausdorff dimension of $X$ cannot be ascertained from finite-dimensional representations of $X$. See $\S 4$ for examples.

$\dagger$ See $\S 2$ for an overview of prevalence. 
The proof of the Banach space theorem uses only the most general information about the structure of the dual space $B^{\prime}$. In specific situations, additional knowledge about the structure of the dual space may yield improved theorems. We show that this does indeed happen in the Hilbert space setting.

Hilbert Space Theorem. Let $H$ be a Hilbert space, and let $M$ be any subspace of the (locally) Lipschitz functions from $H$ to $\mathbb{R}^{m}$ that contains the space of bounded linear functions. Let $X \subset H$ be a compact set with thickness exponent $\tau(X)$. Let $\mu$ be a Borel probability measure supported on $X$. For almost every (in the sense of prevalence) $f \in M$, one has

$$
\operatorname{dim}_{H}(f(X)) \geqslant \min \left\{m, \frac{\operatorname{dim}_{H}(X)}{1+\tau(X) / 2}\right\},
$$

and, for $1<q \leqslant 2$,

$$
D_{q}^{-}(f(\mu)) \geqslant \min \left\{m, \frac{D_{q}^{-}(\mu)}{1+\tau(X) / 2}\right\} .
$$

As we have mentioned, examples in [12] preclude similar results for $0 \leqslant q<1$ and $q>2$. The case $q=1$ is of interest because it corresponds to the commonly used notion of information dimension, in the following sense. In general, the limit (1.1) need not exist. However, $D_{q}^{-}(\mu)$ is a non-increasing function of $q$ and is continuous for $q \neq 1$ [1] . From this it follows that (1.3) and (1.4) hold for $q=1$ if we define

$$
D_{1}^{-}(\mu)=\lim _{q \rightarrow 1^{+}} D_{q}^{-}(\mu) .
$$

Next, we consider the sharpness of the Banach and Hilbert space theorems. In [13], the authors give an example of a compact subset $X$ of Hausdorff dimension $d$ in $\ell^{p}$ for $1 \leqslant p \leqslant \infty$ such that for all bounded linear functions $\pi: \ell^{p} \rightarrow \mathbb{R}^{m}$,

$$
\operatorname{dim}_{\mathrm{H}}(\pi(X)) \leqslant \frac{d}{1+d / q}
$$

where $q=p /(p-1)$. The cases $p=\infty$ and $p=2$ show respectively that the Hausdorff dimension parts of the Banach and Hilbert space theorems are sharp for bounded linear functions on these particular spaces. Notice that $\tau(X)=d$ in these cases.

On the other hand, when $p=1, q$ is infinite, and the example in [13] does not rule out the possibility of a dimension preservation result for subsets of $\ell^{1}$ with positive thickness exponents. We demonstrate that such a result is not possible by constructing a compact subset $X$ of Hausdorff dimension $d$ in $\ell^{1}$ such that, for all bounded linear functions $\pi: \ell^{1} \rightarrow \mathbb{R}^{m}$

$$
\operatorname{dim}_{H}(\pi(X)) \leqslant \frac{d}{1+d / 2} .
$$

In the light of this example, we are somewhat pessimistic regarding the existence of infinite-dimensional spaces for which a general dimension preservation theorem holds. It is thus natural to consider the following fundamental question. Suppose $X$ represents the global attractor of a flow on a function space generated by an evolution equation. Under what hypotheses on the flow does one have $\tau(X)=0$ ? If one assumes that the flow 
is sufficiently dissipative, then $X$ will have finite box-counting dimension. We conjecture that similar dynamical hypotheses imply that $\tau(X)=0$. Friz and Robinson [8] obtain a result of this type. They prove that if an attractor is uniformly bounded in the Sobolev space $H^{s}$ on an appropriate bounded domain in $\mathbb{R}^{m}$, then its thickness exponent is at most $\mathrm{m} / \mathrm{s}$. This result implies that certain attractors of the Navier-Stokes equations have thickness exponent zero. Roughly speaking, thickness is inversely proportional to smoothness.

Section 2 reviews prevalence, the dimension spectrum, and the thickness exponent. The main two theorems are presented and proved in $\S 3$. In $\S 4$ we discuss the sharpness of our results.

\section{Preliminaries}

We discuss prevalence, the dimension spectrum, and the thickness exponent.

2.1. Prevalence. Mathematicians often use topological notions of genericity when formulating theorems in dynamical systems and topology. In topological terms, 'generic' refers to an open and dense subset of mappings, or to a countable intersection of such sets (a 'residual' subset). In finite-dimensional spaces, there exists considerable discord between the topological notion of genericity and the measure-theoretic notion of the size of a set (see $[\mathbf{1 5}, \mathbf{2 3}]$ for examples). Prevalence is intended to be a better analog to 'probability one' on function spaces where no Lebesgue or Haar measure exists.

To motivate the definition of prevalence on a Banach space $B$, consider how the notion of 'Lebesgue almost every' on $\mathbb{R}^{n}$ can be formulated in terms of the same notion on lower-dimensional spaces. Foliate $\mathbb{R}^{n}$ by $k$-dimensional planes, which by an appropriate choice of coordinates we think of as translations of $\mathbb{R}^{k} \subset \mathbb{R}^{n}$ by elements of $\mathbb{R}^{n-k}$. If 'Lebesgue almost every' translation of $\mathbb{R}^{k}$ intersects a Borel set $S \subset \mathbb{R}^{n}$ in full $k$-dimensional Lebesgue measure, then $S$ has full $n$-dimensional Lebesgue measure by the Fubini theorem. If $\mathbb{R}^{n}$ is replaced by an infinite-dimensional space $B$, we cannot formulate the same condition because the space of translations of a $k$-dimensional subspace is infinite-dimensional. However, we can impose the stronger condition that every translation of the subspace intersects $S$ in a set of full Lebesgue measure. A preliminary notion of prevalence is obtained by declaring that a Borel set $S \subset B$ is prevalent if there exists some finite $k$ and some $k$-dimensional subspace $V$ such that every translation of $V$ intersects $S$ in a set of full $k$-dimensional Lebesgue measure. In order to ensure that a countable intersection of prevalent sets is prevalent, we must enlarge the space of measures under consideration beyond Lebesgue measure supported on finite-dimensional subspaces.

Definition 2.1. A Borel set $S \subset B$ is said to be prevalent if there exists a measure $\lambda$ on $B$ such that:

(1) $0<\lambda(C)<\infty$ for some compact subset $C$ of $B$; and

(2) the set $S-x$ has full $\lambda$-measure (that is, the complement of $S-x$ has measure zero) for all $x \in B$.

A non-Borel set that contains a prevalent Borel set is also prevalent. 
The measure $\lambda$ may be a Lebesgue measure on a finite-dimensional subspace of $B$. More generally, one may think of $\lambda$ as describing a family of perturbations in $B$. In this sense, $S$ is prevalent if, for all $x \in B$, choosing a perturbation at random with respect to $\lambda$ and adding it to $x$ yields a point in $S$ with probability one. Prevalent sets share several of the desirable properties of residual sets. A prevalent subset of $B$ is dense and the countable intersection of prevalent sets is prevalent. See [15] for details. One may formulate a notion of prevalence appropriate for spaces without a linear structure [18]. For example, this notion applies to the space of diffeomorphisms of a compact smooth manifold.

2.2. The dimension spectrum. Let $\mu$ be a Borel probability measure on a metric space $X$. For $q \geqslant 0$ and $\epsilon>0$ define

$$
C_{q}(\mu, \epsilon)=\int_{X}[\mu(B(x, \epsilon))]^{q-1} d \mu(x)
$$

where $B(x, \epsilon)$ is the open ball of radius $\epsilon$ centered at $x$.

Definition 2.2. For $q \geqslant 0, q \neq 1$, the lower and upper $q$-dimensions of $\mu$ are

$$
\begin{aligned}
& D_{q}^{-}(\mu)=\liminf _{\epsilon \rightarrow 0} \frac{\log C_{q}(\mu, \epsilon)}{(q-1) \log (\epsilon)}, \\
& D_{q}^{+}(\mu)=\limsup _{\epsilon \rightarrow 0} \frac{\log C_{q}(\mu, \epsilon)}{(q-1) \log (\epsilon)} .
\end{aligned}
$$

If $D_{q}^{-}(\mu)=D_{q}^{+}(\mu)$, their common value is denoted by $D_{q}(\mu)$ and is called the $q$-dimension of $\mu$.

For a measure $\mu$ such that $D_{q}(\mu)$ exists, the function $q \rightarrow D_{q}(\mu)$ is called the dimension spectrum of $\mu$. For $q=0, D_{0}^{-}$and $D_{0}^{+}$depend only on $X$. We write $D_{0}^{-}(X)$ and $D_{0}^{+}(X)$ for the lower and upper zero-dimensions of $X$. For $\epsilon>0$, let $n(X, \epsilon)$ be the minimum number of $\epsilon$-balls required to cover $X$. Written in terms of $n(X, \epsilon), D_{0}^{-}(X)$ and $D_{0}^{+}(X)$ are given by

$$
\begin{aligned}
& D_{0}^{-}(X)=\liminf _{\epsilon \rightarrow 0} \frac{\log n(X, \epsilon)}{\log (1 / \epsilon)}, \\
& D_{0}^{+}(X)=\limsup _{\epsilon \rightarrow 0} \frac{\log n(X, \epsilon)}{\log (1 / \epsilon)} .
\end{aligned}
$$

The values $D_{0}^{-}(X)$ and $D_{0}^{+}(X)$ are therefore equal to the lower and upper box-counting dimensions of $X$, respectively.

For measures on $\mathbb{R}^{n}$, one encounters the following alternative definition of the dimension spectrum $[\mathbf{1 0}, \mathbf{1 1}, \mathbf{2 4}]$. For $\epsilon>0$, cover the support of $\mu$ with a grid of cubes with edge length $\epsilon$. Let $N(\epsilon)$ be the number of cubes that intersect the support of $\mu$, and let the measure of these cubes be $p_{1}, p_{2}, \ldots, p_{N(\epsilon)}$. Write

$$
\begin{aligned}
& D_{q}^{-}(\mu)=\liminf _{\epsilon \rightarrow 0} \frac{\sum_{i=1}^{N(\epsilon)} p_{i}^{q}}{(q-1) \log (\epsilon)}, \\
& D_{q}^{+}(\mu)=\limsup _{\epsilon \rightarrow 0} \frac{\sum_{i=1}^{N(\epsilon)} p_{i}^{q}}{(q-1) \log (\epsilon)} .
\end{aligned}
$$


For $q \geqslant 0, q \neq 1$, these limits are independent of the choice of $\epsilon$-grids, and give the same values as Definition 2.2. See [25] for a proof of this equivalence for $q>1$. The grid definition of the dimension spectrum is not appropriate for measures on general metric spaces. We therefore adopt Definition 2.2 as the natural notion in the general case.

A potential-theoretic definition of the lower $q$-dimension $D_{q}^{-}(\mu)$ for $q>1$ is introduced in [12]. For $s \geqslant 0$ the $s$-potential of the measure $\mu$ at the point $x$ is given by

$$
\varphi_{s}(\mu, x)=\int_{X}|x-y|^{-s} d \mu(y)
$$

Definition 2.3. The $(s, q)$-energy of $\mu$, denoted $I_{s, q}(\mu)$, is given by

$$
I_{s, q}(\mu)=\int_{X}\left[\varphi_{s}(\mu, x)\right]^{q-1} d \mu(x)=\int_{X}\left(\int_{X} \frac{d \mu(y)}{|x-y|^{s}}\right)^{q-1} d \mu(x) .
$$

For $q=2$, the $(s, q)$-energy of $\mu$ reduces to the more standard notion of the $s$-energy of $\mu$, written as

$$
I_{S}(\mu)=\int_{X} \varphi_{S}(\mu, x) d \mu(x)=\int_{X} \int_{X} \frac{d \mu(x) d \mu(y)}{|x-y|^{s}}
$$

Sauer and Yorke [27] show that the lower correlation dimension $D_{2}^{-}(\mu)$ can be expressed as

$$
D_{2}^{-}(\mu)=\sup \left\{s: I_{s}(\mu)<\infty\right\} .
$$

This characterization of $D_{2}^{-}(\mu)$ is used to establish the preservation of correlation dimension. The following proposition generalizes (2.1) to the lower-dimension spectrum for $q>1$.

PROPOSITION 2.4. [12] If $q>1$ and $\mu$ is a Borel probability measure, then

$$
D_{q}^{-}(\mu)=\sup \left\{s \geqslant 0: I_{s, q}(\mu)<\infty\right\} .
$$

2.3. The thickness exponent. Let $B$ denote a Banach space.

Definition 2.5. The thickness exponent $\tau(X)$ of a compact set $X \subset B$ is defined as follows. Let $d(X, \epsilon)$ be the minimum dimension of all finite-dimensional subspaces $V \subset B$ such that every point of $X$ lies within $\epsilon$ of $V$; if no such $V$ exists, then $d(X, \epsilon)=\infty$. Let

$$
\tau(X)=\limsup _{\epsilon \rightarrow 0} \frac{\log d(X, \epsilon)}{\log (1 / \epsilon)} .
$$

There is no general relationship between the thickness exponent and the Hausdorff dimension. A finite-dimensional disk has thickness exponent zero but can have arbitrarily high dimension. A countable set, which necessarily has Hausdorff dimension zero, can have positive thickness exponent. For example, one can show that the compact subset $\left\{0, e_{2} / \log 2, e_{3} / \log 3, \ldots\right\}$ of the real Hilbert space with basis $\left\{e_{1}, e_{2}, \ldots\right\}$ has an infinite thickness exponent. A definitive statement may be made concerning the box-counting dimension $D_{0}$.

Lemma 2.6. [13] Let $X \subset B$ be a compact set. Then $\tau(X) \leqslant D_{0}^{+}(X)$. 
Proof. Recall that the box-counting dimension $D_{0}^{+}(X)$ may be expressed similarly to $\tau(X)$, but in terms of the minimum number $n(X, \epsilon)$ of $\epsilon$-balls required to cover $X$. For any such cover, $X$ lies within $\epsilon$ of the space spanned by the centers of the balls. Thus $d(X, \epsilon) \leqslant n(X, \epsilon)$ for each $\epsilon>0$, and the desired inequality follows.

\section{Main results}

We begin with the main results for general Banach spaces.

THEOREM 3.1. Let $B$ be a Banach space, and let $M$ be any subspace of the Borel measurable functions from $B$ to $\mathbb{R}^{m}$ that contains the bounded linear functions. Let $X \subset B$ be a compact set with thickness exponent $\tau(X)$, and let $\mu$ be a Borel probability measure supported on $X$. For almost every function $f \in M$,

$$
D_{q}^{-}(f(\mu)) \geqslant \min \left\{m, \frac{D_{q}^{-}(\mu)}{1+\tau(X)}\right\}
$$

for all $q \in(1,2]$.

COROllary 3.2. Assume in addition that $M$ is contained in the space of (locally) Lipschitz functions, that $\tau(X)=0$, and that $D_{q}(\mu)$ exists (that is, $D_{q}^{-}(\mu)=D_{q}^{+}(\mu)$ ) for all $q \in(1,2]$. Then for almost every function $f \in M, D_{q}(f(\mu))$ exists and equals $\min \left\{m, D_{q}(\mu)\right\}$ for all $q \in(1,2]$.

Remark 3.3. For $r \geqslant 1$, the space $M=C^{r}\left(B, \mathbb{R}^{m}\right)$ satisfies the hypotheses of Theorem 3.1 and Corollary 3.2.

The corollary follows immediately from Theorem 3.1 and the fact that $D_{q}^{+}(f(\mu)) \leqslant$ $\min \left\{m, D_{q}^{+}(\mu)\right\}$ for all functions $f$ that are Lipschitz on $X$.

COROllary 3.4. Let $B$ be a Banach space. Let $X \subset B$ be a compact set with thickness exponent $\tau(X)$. For almost every function $f \in M$,

$$
\operatorname{dim}_{H}(f(X)) \geqslant \min \left\{m, \frac{\operatorname{dim}_{H}(X)}{1+\tau(X)}\right\} .
$$

Proof. Let $\mathcal{M}(X)$ denote the set of Borel probability measures on $X$. The Hausdorff dimension of $X$ may be expressed in terms of the lower correlation dimension of measures supported on $X$ via the variational principle [5]

$$
\operatorname{dim}_{\mathrm{H}}(X)=\sup _{\mu \in \mathcal{M}(X)} D_{2}^{-}(\mu)
$$

For each $i \in \mathbb{N}$, there exists $\mu_{i} \in \mathcal{M}(X)$ such that $D_{2}^{-}\left(\mu_{i}\right)>\operatorname{dim}_{\mathrm{H}}(X)-1 / i$. Applying Theorem 3.1, there exists a prevalent set $P_{i} \subset M$ of functions such that for $f \in P_{i}$,

$$
D_{2}^{-}\left(f\left(\mu_{i}\right)\right) \geqslant \min \left\{m, \frac{D_{2}^{-}\left(\mu_{i}\right)}{1+\tau(X)}\right\}
$$

The set $\bigcap_{i=1}^{\infty} P_{i}$ is prevalent. For $f \in \bigcap_{i=1}^{\infty} P_{i}$, the bound (3.1) follows from the variational principle. 
Remark 3.5. No analog of Corollary 3.4 holds for the box-counting dimension. Let $n>m$ be integers and let $d \leqslant m$. Sauer and Yorke [27] construct a compact set $A \subset \mathbb{R}^{n}$ such that $D_{0}(A)=d$ and $D_{0}^{+}(f(A))<d$ for every $f \in C^{1}\left(\mathbb{R}^{n}, \mathbb{R}^{m}\right)$.

Proof of Theorem 3.1. Fix $1<q \leqslant 2$. Let $L \subset M$ denote the space of bounded linear functions from $B$ into $\mathbb{R}^{m}$. We will construct a compact 'Banach brick' $Q \subset L$ of perturbations and a probability measure $\lambda$ on $Q$, which will serve as the measure in Definition 2.1 of prevalence. For $f \in M$ and $\pi \in Q$, write $f_{\pi}=f+\pi$. Utilizing the potential-theoretic description of $D_{q}^{-}(\mu)$ for $1<q \leqslant 2$, we will show that for any $f \in M$, $t>0$, and $0 \leqslant s<\min \{m, t /(1+\tau(X))\}$,

$$
I_{t, q}(\mu)<\infty \Rightarrow I_{s, q}\left(f_{\pi}(\mu)\right)<\infty
$$

for $\lambda$-almost every $\pi \in Q$. The theorem will follow because we can choose $t$ arbitrarily close to $D_{q}^{-}(\mu)$.

We define the Banach brick $Q$ and measure $\lambda$ as follows. Think of elements of $L$ as $m$-tuples of elements of the dual space $B^{\prime}$ of $B$. For $j \in \mathbb{N}$, let $d_{j}=d\left(X, 2^{-j}\right)$ and let $V_{j} \subset B$ be a subspace of dimension $d_{j}$ such that every point of $X$ lies within $2^{-j}$ of $V_{j}$. Let $S_{j}$ be the closed unit ball in the dual space $V_{j}^{\prime}$ of $V_{j}$. There is no natural embedding of $V_{j}^{\prime}$ into $B^{\prime}$, but it follows from the Hahn-Banach theorem that there exists an isometric embedding of $V_{j}^{\prime}$ into $B^{\prime}$. As such, we can think of $S_{j}$ as a subset of $B^{\prime}$. On the other hand, $V_{j}^{\prime}$ is linearly isomorphic to $\mathbb{R}^{d_{j}}$, and $S_{j}$ corresponds to a convex set $U_{j} \subset \mathbb{R}^{d_{j}}$. The uniform (Lebesgue) probability measure on $U_{j}$ induces a measure $\lambda_{j}$ on $S_{j}$. Let

$$
Q=\left\{\pi=\left(\pi_{1}, \ldots, \pi_{m}\right): \pi_{i}=\sum_{j=1}^{\infty} j^{-2} \phi_{i j} \text { with } \phi_{i j} \in S_{j} \text { for all } j\right\} .
$$

Since each $S_{j} \subset B^{\prime}$ is compact, $Q \subset L$ is compact. Let $\lambda$ be the probability measure on $Q$ that results from choosing the elements $\phi_{i j}$ randomly and independently with respect to the measures $\lambda_{j}$ on the sets $S_{j}$. (While the term 'brick' suggests that $Q$ is formed from the product of compact sets $j^{-2} S_{j}$ that are all transverse to each other, these sets may have non-trivial intersection, in which case $Q$ and $\lambda$ are still well defined.)

Choose $\rho>\tau(X)$. We will show that, for $0 \leqslant s<m$,

$$
I_{s(1+\rho), q}(\mu)<\infty \Rightarrow I_{s, q}\left(f_{\pi}(\mu)\right)<\infty
$$

for $\lambda$-almost every $\pi \in Q$. Since $\rho$ can be arbitrarily close to $\tau(X)$, this implies (3.2) for fixed $t$. Computing the $(s, q)$-energy of $f_{\pi}(\mu)$, we have

$$
\begin{aligned}
I_{s, q}\left(f_{\pi}(\mu)\right) & =\int_{\mathbb{R}^{m}}\left[\int_{\mathbb{R}^{m}} \frac{d f_{\pi}(\mu)(v)}{|u-v|^{s}}\right]^{q-1} d f_{\pi}(\mu)(u) \\
& =\int_{B}\left[\int_{B} \frac{d \mu(y)}{\left|f_{\pi}(x)-f_{\pi}(y)\right|^{s}}\right]^{q-1} d \mu(x) .
\end{aligned}
$$


Integrating the energy over $Q$ and using the Fubini-Tonelli theorem and the fact that $0<q-1 \leqslant 1$, we have

$$
\begin{aligned}
\int_{Q} I_{s, q}\left(f_{\pi}(\mu)\right) d \lambda(\pi) & =\int_{Q} \int_{B}\left[\int_{B} \frac{d \mu(y)}{\left|f_{\pi}(x)-f_{\pi}(y)\right|^{s}}\right]^{q-1} d \mu(x) d \lambda(\pi) \\
& =\int_{B} \int_{Q}\left[\int_{B} \frac{d \mu(y)}{\left|f_{\pi}(x)-f_{\pi}(y)\right|^{s}}\right]^{q-1} d \lambda(\pi) d \mu(x) \\
& \leqslant \int_{B}\left[\int_{Q} \int_{B} \frac{d \mu(y)}{\left|f_{\pi}(x)-f_{\pi}(y)\right|^{s}} d \lambda(\pi)\right]^{q-1} d \mu(x) \\
& =\int_{B}\left[\int_{B}\left(\int_{Q} \frac{d \lambda(\pi)}{\left|f_{\pi}(x)-f_{\pi}(y)\right|^{s}}\right) d \mu(y)\right]^{q-1} d \mu(x) .
\end{aligned}
$$

The following lemma, which we will prove subsequently, estimates the interior integral.

LEMMA 3.6. (Banach Perturbation Lemma) If $s<m$, there exists a constant $C_{2}$, depending only on $s$ and $\rho$, such that, for all $x, y \in X$,

$$
\int_{Q} \frac{d \lambda(\pi)}{\left|f_{\pi}(x)-f_{\pi}(y)\right|^{s}} \leqslant \frac{C_{2}}{\min \{|x-y|, 1\}^{s(1+\rho)}} .
$$

Applying this lemma to the inequality before it, we have

$$
\int_{Q} I_{s, q}\left(f_{\pi}(\mu)\right) d \lambda(\pi) \leqslant \int_{B}\left[\int_{B} \frac{C_{2}}{\min \{|x-y|, 1\}^{s(1+\rho)}} d \mu(y)\right]^{q-1} d \mu(x) .
$$

Therefore,

$$
I_{s(1+\rho), q}(\mu)<\infty \Rightarrow I_{s, q}\left(f_{\pi}(\mu)\right)<\infty
$$

for $\lambda$-almost every $\pi \in Q$. Since $\rho$ can be arbitrarily close to $\tau(X)$, this implies (3.2) for fixed $t$. Because we can choose $t$ arbitrarily close to $D_{q}^{-}(\mu)$, there exists a prevalent set $P_{q} \subset M$ such that, for $f \in P_{q}$,

$$
D_{q}^{-}(f(\mu)) \geqslant \min \left\{m, \frac{D_{q}^{-}(\mu)}{1+\tau(X)}\right\} .
$$

Let $\left\{q_{i}\right\}$ be a countable dense subset of $(1,2]$. The set $\bigcap_{i=1}^{\infty} P_{q_{i}}$ is prevalent. For $f \in \bigcap_{i=1}^{\infty} P_{q_{i}}$, the continuity of $D_{q}^{-}$on $(1,2]$ implies that

$$
D_{q}^{-}(f(\mu)) \geqslant \min \left\{m, \frac{D_{q}^{-}(\mu)}{1+\tau(X)}\right\}
$$

for all $1<q \leqslant 2$.

Proof of Lemma 3.6. Set $\zeta=\min \{|x-y|, 1\}$. Choose $j \in \mathbb{N}$ such that $3-\log _{2} \zeta \leqslant j \leqslant$ $4-\log _{2} \zeta$. There exist points $\gamma_{j}(x)$ and $\gamma_{j}(y)$ in $V_{j}$ satisfying $\left|\gamma_{j}(x)-x\right| \leqslant 2^{-j}$ and $\left|\gamma_{j}(y)-y\right| \leqslant 2^{-j}$. Since $2^{-j} \leqslant \zeta / 8$, we have

$$
\left|\gamma_{j}(x)-\gamma_{j}(y)\right| \geqslant|x-y|-2 \frac{\zeta}{8} \geqslant \frac{3|x-y|}{4}
$$


Choose $\psi \in S_{j}$ such that $\psi\left(\gamma_{j}(x)-\gamma_{j}(y)\right)=\left|\gamma_{j}(x)-\gamma_{j}(y)\right|$. Recall that $S_{j}$ is the closed unit ball in $V_{j}^{\prime}$, which we embedded isometrically into $B^{\prime}$. It follows that $\left|\psi\left(\gamma_{j}(x)-x\right)\right| \leqslant\left|\gamma_{j}(x)-x\right| \leqslant \zeta / 8$ and $\left|\psi\left(\gamma_{j}(y)-y\right)\right| \leqslant\left|\gamma_{j}(y)-y\right| \leqslant \zeta / 8$, and hence

$$
\psi(x-y) \geqslant \psi\left(\gamma_{j}(x)-\gamma_{j}(y)\right)-2 \frac{\zeta}{8} \geqslant \frac{3|x-y|}{4}-\frac{\zeta}{4} \geqslant \frac{|x-y|}{2} .
$$

For $\pi \in Q$, write $\pi=\xi_{j}+j^{-2} \phi_{j}$ where we define $\phi_{j}=\left(\phi_{1 j}, \ldots, \phi_{m j}\right)$ and $\xi_{j}=\left(\xi_{1 j}, \ldots, \xi_{m j}\right)$ with

$$
\xi_{i j}=\sum_{\substack{k \in \mathbb{N} \\ k \neq j}} k^{-2} \phi_{i k}
$$

for each $i$. Let $\lambda_{j}^{m}$ be the $m$-fold product measure $\lambda_{j} \times \cdots \times \lambda_{j}$ on $S_{j}^{m}=S_{j} \times \cdots \times S_{j} \subset L$. We fix $\xi_{j}$ and integrate $\left|f_{\pi}(x)-f_{\pi}(y)\right|^{s}$ over $\phi_{j} \in S_{j}^{m}$. We have

$$
\begin{aligned}
\int_{S_{j}^{m}} \frac{d \lambda_{j}^{m}\left(\phi_{j}\right)}{\left|f_{\xi_{j}+j^{-2} \phi_{j}}(x)-f_{\xi_{j}+j^{-2} \phi_{j}}(y)\right|^{s}} & =\int_{S_{j}^{m}} \frac{d \lambda_{j}^{m}\left(\phi_{j}\right)}{\left|f_{\xi_{j}}(x)-f_{\xi_{j}}(y)+j^{-2} \phi_{j}(x-y)\right|^{s}} \\
& \leqslant \int_{S_{j}^{m}} \frac{d \lambda_{j}^{m}\left(\phi_{j}\right)}{\left|j^{-2} \phi_{j}(x-y)\right|^{s}} \\
& =j^{2 s} \int_{S_{j}^{m}} \frac{d \lambda_{j}^{m}\left(\phi_{j}\right)}{\left|\phi_{j}(x-y)\right|^{s}} .
\end{aligned}
$$

Let $P \subset B^{\prime}$ be the annihilator of $x-y$. Recall that we have chosen $\psi \in S_{j}$ satisfying (3.3). Since $S_{j}$ is convex, it contains the cone with base $P \cap S_{j}$ and vertex $\psi$. Let $C_{j}$ be this cone, and let $\tilde{\lambda}_{j}$ be the restriction of $\lambda_{j}$ to $C_{j}$, normalized so that $\tilde{\lambda}_{j}\left(C_{j}\right)=1$. We will show that

$$
\int_{S_{j}^{m}} \frac{d \lambda_{j}^{m}\left(\phi_{j}\right)}{\left|\phi_{j}(x-y)\right|^{s}} \leqslant \int_{C_{j}^{m}} \frac{d \tilde{\lambda}_{j}^{m}\left(\phi_{j}\right)}{\left|\phi_{j}(x-y)\right|^{s}} .
$$

Heuristically, this is true because the ball $S_{j}$ is no more concentrated than the cone $C_{j}$ near $P$, where (for each coordinate) the integrand is the largest. To make this statement precise, we foliate $S_{j}^{m}$ and $C_{j}^{m}$ into sets on which $\phi_{j}(x-y)$ is constant.

Let $b=\psi(x-y) \geqslant|x-y| / 2$, and notice that $\phi_{i j}(x-y)$ ranges from 0 to $b$ for $\phi_{i j} \in C_{j}$. Let $g$ be the probability density function of $\left|\phi_{i j}(x-y)\right| / b$ where $\phi_{i j}$ is chosen at random with respect to $\lambda_{j}$, and let $h$ be the probability density function of the same quantity but where $\phi_{i j}$ is chosen at random with respect to $\tilde{\lambda}_{j}$. Then $h$ is supported on $[0,1]$.

Rewriting the integrals in (3.4) using the functions $g$ and $h$, we have

$$
\int_{S_{j}^{m}} \frac{d \lambda_{j}^{m}\left(\phi_{j}\right)}{\left|\phi_{j}(x-y)\right|^{s}}=b^{-s} \int_{0}^{\infty} \cdots \int_{0}^{\infty}|\alpha|^{-s} g\left(\alpha_{1}\right) \cdots g\left(\alpha_{m}\right) d \alpha_{1} \cdots d \alpha_{m}
$$

and

$$
\int_{C_{j}^{m}} \frac{d \tilde{\lambda}_{j}^{m}\left(\phi_{j}\right)}{\left|\phi_{j}(x-y)\right|^{s}}=b^{-s} \int_{0}^{1} \cdots \int_{0}^{1}|\alpha|^{-s} h\left(\alpha_{1}\right) \cdots h\left(\alpha_{m}\right) d \alpha_{1} \cdots d \alpha_{m}
$$


where $\alpha=\left(\alpha_{1}, \ldots, \alpha_{m}\right)$. Therefore, (3.4) is equivalent to

$$
\begin{aligned}
\int_{0}^{\infty} & \cdots \int_{0}^{\infty}|\alpha|^{-s} g\left(\alpha_{1}\right) \cdots g\left(\alpha_{m}\right) d \alpha_{1} \cdots d \alpha_{m} \\
& \leqslant \int_{0}^{1} \cdots \int_{0}^{1}|\alpha|^{-s} h\left(\alpha_{1}\right) \cdots h\left(\alpha_{m}\right) d \alpha_{1} \cdots d \alpha_{m} .
\end{aligned}
$$

We claim that if $k:[0, \infty) \rightarrow \mathbb{R}$ is non-increasing, then

$$
\int_{0}^{1} h(t) k(t) d t \geqslant \int_{0}^{\infty} g(t) k(t) d t
$$

The inequality (3.6) follows from this claim because $|\alpha|^{-s}$ is decreasing in each of its $m$ coordinates.

To verify the claim, recall that we identified $S_{j}$ with a convex set $U_{j} \subset \mathbb{R}^{d_{j}}$. The set $C_{j}$ corresponds to a cone in $U_{j}$. Therefore, $g(t)$ is the suitably normalized $\left(d_{j}-1\right)$ dimensional volume of the slices

$$
\left\{\phi_{i j} \in S_{j}: \phi_{i j}(x-y)= \pm b t\right\} .
$$

Similarly, $h(t)$ is the suitably normalized $\left(d_{j}-1\right)$-dimensional volume of the slice

$$
\left\{\phi_{i j} \in C_{j}: \phi_{i j}(x-y)=b t\right\} .
$$

The functions are each normalized so that $\int_{0}^{\infty} g(t) d t=\int_{0}^{1} h(t) d t=1$. The slices of the cone $C_{j}$ are parallel to its base, and thus all have the same shape. Since $t=0$ corresponds to the base and $t=1$ corresponds to the vertex, $h(t)$ is proportional to $(1-t)^{d_{j}-1}$ for $t \in[0,1]$, and $h(t)=0$ for $t>1$. By symmetry, the slices of $S_{j}$ on which $\phi_{i j}(x-y)=-b t$ and on which $\phi_{i j}(x-y)=b t$ have the same $\left(d_{j}-1\right)$ dimensional volume, so $g(t)$ is proportional to the volume of the latter slice. Since $S_{j}$ is convex, $g^{1 /\left(d_{j}-1\right)}$ is concave on the interval that supports $g$ by the Brunn-Minkowski inequality [9]. This interval contains $[0,1]$ because $S_{j}$ contains $C_{j}$. Then since $h^{1 /\left(d_{j}-1\right)}$ is linear on $[0,1]$ and $g(1) \geqslant 0=h(1)$, if $g(t)<h(t)$ for some $t \in(0,1)$ then $g<h$ on $[0, t]$. Let $c$ be the least number in $[0,1]$ for which $g(c) \geqslant h(c)$; then $g(t)<h(t)$ for $0 \leqslant t<c$ and $g(t) \geqslant h(t)$ for $t \geqslant c$. Finally, since $k$ is non-increasing,

$$
\begin{aligned}
\int_{0}^{c}(h(t)-g(t)) k(t) d t & \geqslant k(c) \int_{0}^{c}(h(t)-g(t)) d t \\
& =k(c) \int_{c}^{\infty}(g(t)-h(t)) d t \\
& \geqslant \int_{c}^{\infty}(g(t)-h(t)) k(t) d t
\end{aligned}
$$

which is equivalent to (3.7).

We now need to estimate the integral on the right-hand side of (3.5); this is not hard because we know $h$ explicitly.

LEMMA 3.7. (Banach integral asymptotics) Let $m \in \mathbb{N}$ and $s<m$. There exists a constant $K$, independent of $n \in \mathbb{N}$, such that

$$
\frac{\int_{0}^{1} \cdots \int_{0}^{1}|\alpha|^{-s}\left(1-\alpha_{1}\right)^{n-1} \cdots\left(1-\alpha_{m}\right)^{n-1} d \alpha_{1} \cdots d \alpha_{m}}{\int_{0}^{1} \cdots \int_{0}^{1}\left(1-\alpha_{1}\right)^{n-1} \cdots\left(1-\alpha_{m}\right)^{n-1} d \alpha_{1} \cdots d \alpha_{m}} \leqslant K n^{s},
$$

where $\alpha=\left(\alpha_{1}, \ldots, \alpha_{m}\right)$. 
Proof. Since $e^{-z} \geqslant 1-z$ for all real $z$, and the denominator of (3.8) is $n^{-m}$, the ratio of integrals in (3.8) is bounded above by

$$
n^{m} \int_{0}^{\infty} \cdots \int_{0}^{\infty} \frac{\exp \left(-\sum_{i=1}^{m} \alpha_{i}(n-1)\right)}{|\alpha|^{s}} d \alpha_{1} \cdots d \alpha_{m} .
$$

Setting $u_{i}=\alpha_{i}(n-1)$, this becomes

$$
n^{m}(n-1)^{s-m} \int_{0}^{\infty} \cdots \int_{0}^{\infty} \frac{\exp \left(-\sum_{i=1}^{m} u_{i}\right)}{|u|^{s}} d u_{1} \cdots d u_{m} .
$$

Since $|u|^{-s}$ is integrable in a neighborhood of zero for $s<m$, the lemma is established.

We now complete the proof of Lemma 3.6. Recall that $d_{j}=d\left(X, 2^{-j}\right)$ is the dimension of $V_{j}$. Let $\sigma=(\rho+\tau(X)) / 2$, so that $\rho>\sigma>\tau(X)$. By Definition 2.5 of $\tau(X)$, there exists $C_{1}>0$, depending only on $X$ and $\sigma$, such that $d_{j} \leqslant C_{1} 2^{j \sigma}$. It follows from (3.4), (3.5), and Lemma 3.7 with $n=d_{j} \leqslant C_{1} 2^{j \sigma}$ that there exists $K$, independent of $j$, such that

$$
\begin{aligned}
j^{2 s} \int_{S_{j}^{m}} \frac{d \lambda_{j}^{m}\left(\phi_{j}\right)}{\left|\phi_{j}(x-y)\right|^{s}} & \leqslant j^{2 s} b^{-s} K\left(C_{1} 2^{j \sigma}\right)^{s} \\
& \leqslant K C_{1}^{s} j^{2 s} 2^{s}|x-y|^{-s}\left(2^{j}\right)^{\sigma s} \\
& \leqslant K C_{1}^{s}\left(4-\log _{2} \zeta\right)^{2 s} 2^{s}|x-y|^{-s}\left(16 \zeta^{-1}\right)^{\sigma s} \\
& \leqslant 2^{(1+4 \sigma) s} K C_{1}^{s}\left(4-\log _{2} \zeta\right)^{2 s} \zeta^{-s(1+\sigma)}
\end{aligned}
$$

Thus, since $\rho>\sigma$, there exists $C_{2}$ such that

$$
j^{2 s} \int_{S_{j}^{m}} \frac{d \lambda_{j}^{m}\left(\phi_{j}\right)}{\left|\phi_{j}(x-y)\right|^{s}} \leqslant \frac{C_{2}}{\zeta^{s(1+\rho)}} .
$$

We have established that

$$
\int_{S_{j}^{m}} \frac{d \lambda_{j}^{m}\left(\phi_{j}\right)}{\left|f_{\xi_{j}+j^{-2} \phi_{j}}(x)-f_{\xi_{j}+j^{-2} \phi_{j}}(y)\right|^{s}} \leqslant \frac{C_{2}}{\zeta^{s(1+\rho)}}
$$

for all $\xi_{j}$, and hence by integrating over $\xi_{j}$ that

$$
\int_{Q} \frac{d \lambda(\pi)}{\left|f_{\pi}(x)-f_{\pi}(y)\right|^{s}} \leqslant \frac{C_{2}}{\zeta^{s(1+\rho)}} .
$$

The proof of Lemma 3.6 is complete.

The proof of this perturbation lemma uses only the convexity of $S_{j}$. In specific cases, additional information about the geometry of the unit ball in the dual space may lead to an improved perturbation lemma, and hence to an improvement of the factor $1 /(1+\tau(X))$. We establish such an improvement for Hilbert spaces.

THEOREM 3.8. Let $H$ be a Hilbert space, and let $M$ be any subspace of the Borel measurable functions from $H$ to $\mathbb{R}^{m}$ that contains the bounded linear functions. 
Let $X \subset H$ be a compact set with thickness exponent $\tau(X)$, and let $\mu$ be a Borel probability measure supported on $X$. For almost every function $f \in M$,

$$
D_{q}^{-}(f(\mu)) \geqslant \min \left\{m, \frac{D_{q}^{-}(\mu)}{1+\tau(X) / 2}\right\}
$$

for all $q \in(1,2]$.

COROLlary 3.9. Let $H$ be a Hilbert space. Let $X \subset H$ be a compact set with thickness exponent $\tau(X)$. For almost every function $f \in M$,

$$
\operatorname{dim}_{H}(f(X)) \geqslant \min \left\{m, \frac{\operatorname{dim}_{H}(X)}{1+\tau(X) / 2}\right\} .
$$

Remark 3.10. For the example from [13] discussed in the introduction, this Hausdorff dimension estimate is sharp.

Proof of Theorem 3.8. Let $L \subset M$ denote the space of bounded linear functions from $H$ into $\mathbb{R}^{m}$. As in the proof of Theorem 3.1, we construct a probability measure $\lambda$ on a compact subset $Q \subset L$ such that for any $f \in M, t>0$, and $0 \leqslant s<$ $\min \{m, t /(1+\tau(X) / 2)\}$,

$$
I_{t, q}(\mu)<\infty \Rightarrow I_{s, q}\left(f_{\pi}(\mu)\right)<\infty
$$

for $\lambda$-almost every $\pi \in Q$. The construction of the Hilbert brick $Q$ follows that of the Banach brick. Notice that each $S_{j}$ is isometric to a Euclidean ball. The dual space $V_{j}^{\prime}$ embeds canonically into $H^{\prime}=H$ : an element of $V_{j}^{\prime}$ acts on an element of $H$ by composition with the orthogonal projection onto $V_{j}$. Choose $\rho>\tau(X)$. We will show that for $0 \leqslant s<m$,

$$
I_{s(1+\rho / 2), q}(\mu)<\infty \Rightarrow I_{s, q}\left(f_{\pi}(\mu)\right)<\infty
$$

for $\lambda$-almost every $\pi \in Q$. The proof of this implication follows closely the argument given in the proof of Theorem 3.1. We only need to apply the following improved perturbation lemma.

Lemma 3.11. (Hilbert Perturbation Lemma) If $s<m$, there exists a constant $C_{3}$, depending only on $s$ and $\rho$, such that, for all $x, y \in X$,

$$
\int_{Q} \frac{d \lambda(\pi)}{\left|f_{\pi}(x)-f_{\pi}(y)\right|^{s}} \leqslant \frac{C_{3}}{\min \{|x-y|, 1\}^{s(1+\rho / 2)}} .
$$

Proof. Set $\zeta=\min \{|x-y|, 1\}$. Select $j$ and decompose $\pi=\xi_{j}+j^{-2} \phi_{j}$ as in the proof of Lemma 3.6; as before we have

$$
\int_{S_{j}^{m}} \frac{d \lambda_{j}^{m}\left(\phi_{j}\right)}{\left|f_{\xi_{j}+j^{-2} \phi_{j}}(x)-f_{\xi_{j}+j^{-2} \phi_{j}}(y)\right|^{s}} \leqslant j^{2 s} \int_{S_{j}^{m}} \frac{d \lambda_{j}^{m}\left(\phi_{j}\right)}{\left|\phi_{j}(x-y)\right|^{s}} .
$$

Since we are now in a Hilbert space, we can simply let $\gamma_{j}(x)$ and $\gamma_{j}(y)$ be the orthogonal projections of $x$ and $y$ onto $V_{j}$. Then for $\phi_{j} \in S_{j}^{m}$, we have $\phi_{j}(x-y)=\phi_{j}\left(\gamma_{j}(x)-\gamma_{j}(y)\right)$. Each coordinate $\phi_{i j}(x-y)$ thus ranges between $-\left|\gamma_{j}(x)-\gamma_{j}(y)\right|$ and $\left|\gamma_{j}(x)-\gamma_{j}(y)\right| \geqslant$ $3|x-y| / 4$. If $\phi_{i j}$ is distributed according to the (uniform) measure $\lambda_{j}$ on $S_{j}$, then as before 
we can express the probability density function of $\phi_{i j}(x-y) /\left|\gamma_{j}(x)-\gamma_{j}(y)\right|$ in terms of the $\left(d_{j}-1\right)$-dimensional volumes of parallel slices of $S_{j}$. Since $S_{j}$ is a Euclidean ball, we know the volumes of these slices. Thus, we can estimate the integral over $S_{j}^{m}$ above directly, without inscribing a cone in $S_{j}$. We need the following analog of Lemma 3.7.

LEMmA 3.12. (Hilbert integral asymptotics) There exists $K>0$, independent of $n \in \mathbb{N}$, such that, for $s<m$,

$$
\frac{\int_{0}^{1} \cdots \int_{0}^{1}|\alpha|^{-s}\left(1-\alpha_{1}^{2}\right)^{(n-1) / 2} \cdots\left(1-\alpha_{m}^{2}\right)^{(n-1) / 2} d \alpha_{1} \cdots d \alpha_{m}}{\int_{0}^{1} \cdots \int_{0}^{1}\left(1-\alpha_{1}^{2}\right)^{(n-1) / 2} \cdots\left(1-\alpha_{m}^{2}\right)^{(n-1) / 2} d \alpha_{1} \cdots d \alpha_{m}} \leqslant K n^{s / 2} .
$$

Proof. The proof is similar to that of Lemma 3.7 and is left to the reader.

As before, letting $\sigma=(\rho+\tau(X)) / 2$ and using Lemma 3.12 with $n=d_{j} \leqslant C_{1} 2^{j \sigma}$, we have

$$
\begin{aligned}
j^{2 s} \int_{S_{j}^{m}} \frac{d \lambda_{j}^{m}\left(\phi_{j}\right)}{\left|\phi_{j}(x-y)\right|^{s}} & \leqslant j^{2 s}\left|\gamma_{j}(x)-\gamma_{j}(y)\right|^{-s} K\left(C_{1} 2^{j \sigma}\right)^{s / 2} \\
& \leqslant K C_{1}^{s / 2} j^{2 s}(4 / 3)^{s}|x-y|^{-s}\left(2^{j}\right)^{\sigma s / 2} \\
& \leqslant(4 / 3)^{s} K C_{1}^{s / 2}\left(4-\log _{2} \zeta\right)^{2 s}|x-y|^{-s}\left(16 \zeta^{-1}\right)^{\sigma s / 2} \\
& \leqslant(4 / 3)^{s} 16^{\sigma s / 2} K C_{1}^{s / 2}\left(4-\log _{2} \zeta\right)^{2 s} \zeta^{-s(1+\sigma / 2)} \\
& \leqslant C_{3} \zeta^{-s(1+\rho / 2)}
\end{aligned}
$$

for some $C_{3}>0$. The rest of the proof is analogous to that of Lemma 3.6.

\section{Non-preservation of Hausdorff dimension}

Theorems 3.1 and 3.8 are sharp in the following sense. Given $d>0,1 \leqslant p \leqslant \infty$, and a positive integer $m$, there is a compact subset $X$ of Hausdorff dimension $d$ in $\ell^{p}$ such that, for all bounded linear functions $\pi: \ell^{p} \rightarrow \mathbb{R}^{m}$,

$$
\operatorname{dim}_{H}(\pi(X)) \leqslant \frac{d}{1+d / q}
$$

where $q=p /(p-1)$ [13]. (The case $p=\infty$ was excluded in [13] because the dual space of $\ell^{\infty}$ is not $\ell^{1}$, but the argument there carries over to this case for the following reason. In this case, $X$ lies in the space $c_{0} \subset \ell^{\infty}$ of sequences that converge to zero. Since the dual space of $c_{0}$ is $\ell^{1}$, every element of the dual space of $\ell^{\infty}$ acts on $X$ like an element of $\ell^{1}$.) The cases $p=\infty$ and $p=2$ show respectively that Theorems 3.1 and 3.8 are sharp for bounded linear functions on these particular Banach spaces. On the other hand, this class of examples does not rule out a dimension preservation result in $\ell^{1}$. The following example does.

Here we construct a different compact subset $X$ of Hausdorff dimension $d$ in $\ell^{1}$ such that, for all bounded linear functions $\pi: \ell^{1} \rightarrow \mathbb{R}^{m}$,

$$
\operatorname{dim}_{H}(\pi(X)) \leqslant \frac{d}{1+d / 2} .
$$




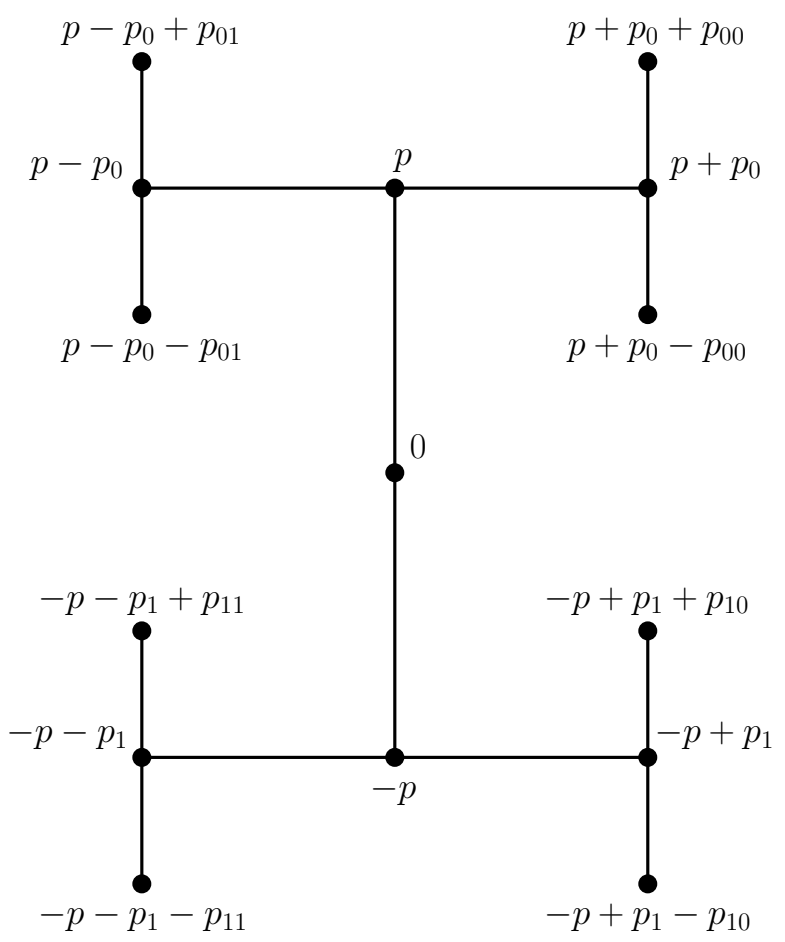

FIGURE 1 . The sets $X_{0}, X_{1}, X_{2}$, and $X_{3}$ consist of the nodes of the binary tree above.

Let $\left\{e_{1}, e_{2}, \ldots\right\}$ be the standard basis of $\ell^{1}$, and let $\lambda=2^{-1 / d}$. Consider the inductively constructed sets $X_{k}$, defined as follows. Each $X_{k}$ will have $2^{k}$ points, which we think of as nodes at the $k$ th level of a binary tree (see Figure 1). Let $X_{0}=\{0\}$ and $X_{1}=\{ \pm p\}$, where

$$
p=\frac{1}{2}\left(e_{1}-e_{2}\right)
$$

For the next step, construct the two points

$$
p_{0}=\frac{\lambda}{4}\left(e_{3}-e_{4}+e_{5}-e_{6}\right), \quad p_{1}=\frac{\lambda}{4}\left(e_{3}+e_{4}-e_{5}-e_{6}\right) .
$$

Add $\pm p_{0}$ to one point of $X_{1}$ and $\pm p_{1}$ to the other, forming the set

$$
X_{2}=\left\{p \pm p_{0},-p \pm p_{1}\right\} .
$$

We now describe the construction of $X_{k+1}$ given $X_{k}$ of the form

$$
X_{k}=\left\{(-1)^{\omega_{1}} p+(-1)^{\omega_{2}} p_{\omega_{1}}+\cdots+(-1)^{\omega_{k}} p_{\omega_{1} \cdots \omega_{k-1}}: \omega_{1}, \ldots, \omega_{k} \in\{0,1\}\right\} .
$$

Let

$$
\alpha_{k}=1+\sum_{j=0}^{k-1} 2^{2^{j}}
$$

be the smallest index for which $e_{\alpha_{k}}$ is not used in the definition of $X_{k}$. Define the collection of $2^{k}$ points

$$
\left\{p_{\omega_{1} \omega_{2} \cdots \omega_{k}}: \omega_{1}, \omega_{2}, \ldots, \omega_{k} \in\{0,1\}\right\}
$$


by setting

$$
p_{\omega_{1} \omega_{2} \cdots \omega_{k}}=\frac{\lambda^{k}}{2^{2^{k}}} \sum_{j=0}^{2^{2^{k}}-1}(-1)^{\left\lfloor j / 2^{\gamma \omega_{1} \cdots \omega_{k}}\right\rfloor} e_{\alpha_{k}+j},
$$

where $\gamma_{\omega_{1} \cdots \omega_{k}}$ is the integer in $\left[0,2^{k}\right)$ whose binary representation is $\omega_{1} \cdots \omega_{k}$; that is,

$$
\gamma_{\omega_{1} \cdots \omega_{k}}=\omega_{1} 2^{k-1}+\omega_{2} 2^{k-2}+\cdots+\omega_{k} .
$$

Notice that $\left\|p_{\omega_{1} \cdots \omega_{k}}\right\|_{\ell^{1}}=\lambda^{k}$. Add $\pm p_{\omega_{1} \cdots \omega_{k}}$ to the corresponding point of $X_{k}$, forming

$$
X_{k+1}=\left\{(-1)^{\omega_{1}} p+(-1)^{\omega_{2}} p_{\omega_{1}}+\cdots+(-1)^{\omega_{k+1}} p_{\omega_{1} \cdots \omega_{k}}: \omega_{1}, \ldots, \omega_{k+1} \in\{0,1\}\right\} .
$$

Remark 4.1. The reason for choosing the points $p_{\omega_{1} \omega_{2} \cdots \omega_{k}}$ in this manner is so that each element $\xi$ of the dual space $\ell^{\infty}$ of $\ell^{1}$ must come close to annihilating most of these points. For example, if $\xi_{j}=(-1)^{j+1}$, then $\xi\left(p_{00}\right)=\lambda^{2}$ but $\xi\left(p_{01}\right)=\xi\left(p_{10}\right)=\xi\left(p_{11}\right)=0$. Lemma 4.4 below says that no other choice of $\xi$ with the same norm yields a larger meansquare average of $\xi\left(p_{\omega_{1} \omega_{2}}\right)$.

Figure 1 illustrates the third step in the construction, where

$$
\begin{aligned}
p_{00}= & \frac{\lambda^{2}}{16}\left(e_{7}-e_{8}+e_{9}-e_{10}+e_{11}-e_{12}+e_{13}-e_{14}\right. \\
& \left.+e_{15}-e_{16}+e_{17}-e_{18}+e_{19}-e_{20}+e_{21}-e_{22}\right), \\
p_{01}= & \frac{\lambda^{2}}{16}\left(e_{7}+e_{8}-e_{9}-e_{10}+e_{11}+e_{12}-e_{13}-e_{14}\right. \\
& \left.+e_{15}+e_{16}-e_{17}-e_{18}+e_{19}+e_{20}-e_{21}-e_{22}\right), \\
p_{10}= & \frac{\lambda^{2}}{16}\left(e_{7}+e_{8}+e_{9}+e_{10}-e_{11}-e_{12}-e_{13}-e_{14}\right. \\
& \left.+e_{15}+e_{16}+e_{17}+e_{18}-e_{19}-e_{20}-e_{21}-e_{22}\right), \\
& \frac{\lambda^{2}}{16}\left(e_{7}+e_{8}+e_{9}+e_{10}+e_{11}+e_{12}+e_{13}+e_{14}\right. \\
& \left.-e_{15}-e_{16}-e_{17}-e_{18}-e_{19}-e_{20}-e_{21}-e_{22}\right) .
\end{aligned}
$$

Let $X$ be the set of all limit points of $X_{0} \cup X_{1} \cup X_{2} \cup \ldots$. Equivalently,

$$
X=\left\{(-1)^{\omega_{1}} p+(-1)^{\omega_{2}} p_{\omega_{1}}+(-1)^{\omega_{3}} p_{\omega_{1} \omega_{2}}+\cdots: \omega_{1}, \omega_{2}, \omega_{3}, \cdots \in\{0,1\}\right\} .
$$

Notice that each element of $X$ has $\ell^{1}$ norm $1+\lambda+\lambda^{2}+\cdots=1 /(1-\lambda)$.

Proposition 4.2. For the set $X \subset \ell^{1}$ constructed above,

$$
\operatorname{dim}_{\mathrm{H}}(X)=D_{0}^{+}(X)=\frac{\log 2}{\log (1 / \lambda)}=d .
$$

Proof. The set $X$ can be covered by $2^{k}$ balls of radius $\lambda^{k} /(1-\lambda)$ centered at the points of $X_{k}$, so $\operatorname{dim}_{H}(X) \leqslant D_{0}^{+}(X) \leqslant d$. To show that $\operatorname{dim}_{H}(X) \geqslant d$, we apply Frostman's lemma [5, 22]. Identify $X$ with the set of binary strings $S=\left\{\omega=\omega_{1} \omega_{2} \omega_{3} \cdots: \omega_{1}, \omega_{2}\right.$, $\left.\omega_{3}, \cdots \in\{0,1\}\right\}$. Consider the measure $\mu$ on $X$ induced by the uniform probability 
measure on $S$. Since every two points in $X$ corresponding to different initial strings $\omega_{1} \cdots \omega_{k} \omega_{k+1}$ and $\omega_{1} \cdots \omega_{k} \omega_{k+1}^{\prime}$ must lie at least $2 \lambda^{k}$ apart, the measure of a ball of radius less than $\lambda^{k}$ is at most the measure of all strings in $S$ starting with a given $\omega_{1} \cdots \omega_{k+1}$, which is $2^{-(k+1)}=\left(\lambda^{k}\right)^{d} / 2$. By Frostman's lemma, $\operatorname{dim}_{\mathrm{H}}(X) \geqslant d$.

Proposition 4.3. Fix $m \in \mathbb{N}$. For every bounded linear map $\pi: \ell^{1} \rightarrow \mathbb{R}^{m}$,

$$
\operatorname{dim}_{H}(\pi(X)) \leqslant \frac{d}{1+d / 2}
$$

Proof. Let $s=d /(1+d / 2)=(1 / d+1 / 2)^{-1}$. Let $\pi: \ell^{1} \rightarrow \mathbb{R}^{m}$ be a bounded linear map and assume $\|\pi\|=1$. We will show that, for each $k \geqslant 0, \pi(X)$ can be covered by a collection of $2^{k}$ sets

$$
\mathcal{E}_{k}=\left\{E_{i}: i=0, \ldots, 2^{k}-1\right\}
$$

such that

$$
\lim _{k \rightarrow \infty} \max _{E \in \mathcal{E}_{k}} \operatorname{diam}(E)=0
$$

and

$$
\sum_{i=0}^{2^{k}-1} \operatorname{diam}\left(E_{i}\right)^{s}
$$

remains bounded as $k \rightarrow \infty$. It then follows that the $s$-dimensional Hausdorff measure of $\pi(X)$ is finite, and therefore that the Hausdorff dimension of $\pi(X)$ is at most $s$, as desired. Since $s<2$, by convexity

$$
2^{-k} \sum_{i=0}^{2^{k}-1}\left(\operatorname{diam}\left(E_{i}\right)^{2}\right)^{s / 2} \leqslant\left(2^{-k} \sum_{i=0}^{2^{k}-1} \operatorname{diam}\left(E_{i}\right)^{2}\right)^{s / 2} .
$$

Therefore, it suffices to show that

$$
2^{-k} \sum_{i=0}^{2^{k}-1} \operatorname{diam}\left(E_{i}\right)^{2} \leqslant C_{4} 2^{-2 k / s}=C_{4} 2^{-k} \lambda^{2 k}
$$

for some constant $C_{4}$ independent of $k$. Each set $E_{i}$ will cover the image under $\pi$ of the part $P_{i}$ of $X$ corresponding to the $i$ th point in $X_{k}$. The diameters of the $E_{i}$ then depend on how $\pi$ acts on the branches of levels $k+1, k+2, \ldots$ of the tree used to form $X$. First we consider how a single coordinate $\xi$ of $\pi$ acts on level $k+1$ of this tree.

Recall that

$$
N_{k}=\left\{(-1)^{\omega_{k+1}} p_{\omega_{1} \cdots \omega_{k}}: \omega_{1}, \ldots, \omega_{k+1} \in\{0,1\}\right\}
$$

is the set of points used to perturb the $2^{k}$ points of $X_{k}$ to form the $2^{k+1}$ points of $X_{k+1}$. We seek an asymptotic bound on the quantity

$$
Z_{k}=\sup _{\|\xi\|_{\ell} \infty=1} \sum_{r \in N_{k}} \xi(r)^{2} .
$$

LEMMA 4.4. We have $Z_{k} \leqslant 2 \lambda^{2 k}$. 
Proof. For each $\omega_{1} \cdots \omega_{k} \in\{0,1\}^{k}, N_{k}$ contains $p_{\omega_{1} \cdots \omega_{k}}$ and $-p_{\omega_{1} \cdots \omega_{k}}$. Define

$$
N_{k}^{+}=\left\{p_{\omega_{1} \cdots \omega_{k}}: \omega_{1}, \ldots, \omega_{k} \in\{0,1\}\right\}
$$

We reindex the elements of $N_{k}^{+}$by $\gamma_{\omega_{1} \cdots \omega_{k}}$, obtaining $N_{k}^{+}=\left\{p_{i}: i=0, \ldots, 2^{k}-1\right\}$. By symmetry, $Z_{k}=2 Z_{k}^{+}$, where

$$
Z_{k}^{+}=\sup _{\|\xi\|_{\ell} \infty=1} \sum_{r \in N_{k}^{+}} \xi(r)^{2} .
$$

Think of the points of $N_{k}^{+}$as the rows of a $2^{k} \times 2^{2^{k}}$ matrix. The entry in row $i$, column $j$ of this matrix (starting the numbering at $i=0$ and $j=0$ ) is the coefficient of $e_{\alpha_{k}+j}$ in $p_{i}$, namely

$$
p_{i j}=\frac{\lambda^{k}}{2^{2^{k}}}(-1)^{\left\lfloor j / 2^{i}\right\rfloor} .
$$

Let $\left(s_{i j}\right)$ be the associated matrix of signs, defined by

$$
s_{i j}=(-1)^{\left\lfloor j / 2^{i}\right\rfloor} .
$$

Let $\left\{Y_{i}: i=0, \ldots, 2^{k}-1\right\}$ be a collection of independent Bernoulli random variables, each taking the value 1 with probability $1 / 2$ and the value -1 with probability $1 / 2$. The columns $\boldsymbol{s}_{j}$ of $\left(s_{i j}\right)$ constitute the sample space of the random vector $\boldsymbol{Y}=\left(Y_{0}, Y_{1}, \ldots, Y_{2^{k}-1}\right)$. In terms of $\boldsymbol{Y}$, we have

$$
\sup _{\|\xi\|_{\ell^{\infty}=1}} \sum_{r \in N_{k}^{+}} \xi(r)^{2}=\left(\frac{\lambda^{k}}{2^{2^{k}}}\right)^{2} \sup _{\|\xi\|_{\ell^{\infty}=1}}\left|\sum_{j=0}^{2^{2^{k}}-1} \xi_{\alpha_{k}+j} s_{j}\right|^{2} .
$$

Since

$$
\left|\sum_{j=0}^{2^{2^{k}}-1} \xi_{\alpha_{k}+j} \boldsymbol{s}_{j}\right|=\max _{|\boldsymbol{b}|=1}\left\langle\boldsymbol{b}, \sum_{j=0}^{2^{2^{k}}-1} \xi_{\alpha_{k}+j} \boldsymbol{s}_{j}\right\rangle,
$$

we have

$$
\begin{aligned}
\left(\frac{\lambda^{k}}{2^{2^{k}}}\right)^{2} \sup _{\|\xi\|_{\ell} \infty=1}\left|\sum_{j=0}^{2^{2^{k}}-1} \xi_{\alpha_{k}+j} \boldsymbol{s}_{j}\right|^{2} & =\left(\frac{\lambda^{k}}{2^{2^{k}}}\right)^{2} \sup _{\|\xi\|_{\ell}=1} \max _{|\boldsymbol{b}|=1}\left\langle\boldsymbol{b}, \sum_{j=0}^{2^{2^{k}}-1} \xi_{\alpha_{k}+j} \boldsymbol{s}_{j}\right\rangle^{2} \\
& \leqslant\left(\frac{\lambda^{k}}{2^{2^{k}}}\right)^{2}\left(2^{2^{k}}\right)^{2} \max _{|\boldsymbol{b}|=1} E[|\boldsymbol{b} \cdot \boldsymbol{Y}|]^{2} \\
& \leqslant \lambda^{2 k} \max _{|\boldsymbol{b}|=1} \operatorname{Var}[\boldsymbol{b} \cdot \boldsymbol{Y}] \\
& =\lambda^{2 k}
\end{aligned}
$$

Therefore, $Z_{k}^{+} \leqslant \lambda^{2 k}$, and we conclude that $Z_{k}=2 Z_{k}^{+} \leqslant 2 \lambda^{2 k}$.

Returning to the proof of Proposition 4.3, we show that, for each $k \geqslant 0, \pi(X)$ can be covered by $2^{k}$ sets $E_{0}, \ldots, E_{2^{k}-1}$ such that

$$
\sum_{i=0}^{2^{k}-1} \operatorname{diam}\left(E_{i}\right)^{2} \leqslant C_{4} \lambda^{2 k}
$$


for some constant $C_{4}$ independent of $k$. Fix $k \geqslant 0$. For each string $\omega_{1} \cdots \omega_{k}$, let $P_{\omega_{1} \cdots \omega_{k}}$ denote the subtree

$$
\begin{aligned}
\left\{(-1)^{\omega_{1}} p+(-1)^{\omega_{2}} p_{\omega_{1}}+\cdots\right. & +(-1)^{\omega_{k}} p_{\omega_{1} \cdots \omega_{k-1}}+(-1)^{\omega_{k+1}} p_{\omega_{1} \cdots \omega_{k}} \\
& \left.+(-1)^{\omega_{k+2}} p_{\omega_{1} \cdots \omega_{k+1}}+\cdots: \omega_{k+1}, \omega_{k+2}, \cdots \in\{0,1\}\right\} .
\end{aligned}
$$

In terms of binary strings, $P_{\omega_{1} \cdots \omega_{k}}$ corresponds to the collection

$$
S_{\omega_{1} \cdots \omega_{k}}=\left\{\beta \in\{0,1\}^{\mathbb{N}}: \beta_{1} \cdots \beta_{k}=\omega_{1} \cdots \omega_{k}\right\} .
$$

The image $\pi\left(P_{\omega_{1} \cdots \omega_{k}}\right)$ can be covered by a ball $E_{i}=E_{\gamma_{\omega_{1} \cdots \omega_{k}}}$ centered at

$$
\kappa_{i}=\pi\left((-1)^{\omega_{1}} p+(-1)^{\omega_{2}} p_{\omega_{1}}+\cdots+(-1)^{\omega_{k}} p_{\omega_{1} \cdots \omega_{k-1}}\right)
$$

of radius

$$
\sup _{\rho \in P_{\omega_{1} \cdots \omega_{k}}}\left|\pi(\rho)-\kappa_{i}\right| .
$$

The collection $\left\{E_{i}: i=0, \cdots, 2^{k}-1\right\}$ therefore covers $\pi(X)$. We have

$$
\sum_{i=0}^{2^{k}-1} \operatorname{diam}\left(E_{i}\right)^{2} \leqslant 4 \sum_{i=0}^{2^{k}-1} \max _{\rho \in P_{\omega_{1} \cdots \omega_{k}}}\left|\sum_{j=k+1}^{\infty} \pi\left(\rho_{j}\right)\right|^{2},
$$

where if $\rho \in P_{\omega_{1} \cdots \omega_{k}}$ corresponds to string $\beta \in\{0,1\}^{\mathbb{N}}$, then $\rho_{j}=(-1)^{\beta_{j}} p_{\beta_{1} \ldots \beta_{j-1}}$. In order to bound the right-hand side of (4.1), we need the following lemma.

LEMMA 4.5. Let $\left\{v_{j}: 0, \ldots, \infty\right\}$ be a collection of vectors in $\mathbb{R}^{m}$ and let $0<\chi<1$. We have

$$
\left|\sum_{j=0}^{\infty} v_{j}\right|^{2} \leqslant \frac{1}{1-\chi} \sum_{j=0}^{\infty} \chi^{-j}\left|v_{j}\right|^{2} .
$$

Proof. If the right-hand side of the inequality is infinite, the inequality is trivially true. Otherwise, $v_{j}$ must decay exponentially as $j$ increases, and all the sums below converge absolutely. Using the Cauchy-Schwartz inequality,

$$
\begin{aligned}
\left|\sum_{j=0}^{\infty} v_{j}\right|^{2} & =\left\langle\sum_{j=0}^{\infty} v_{j}, \sum_{i=0}^{\infty} v_{i}\right\rangle \\
& =\sum_{j=0}^{\infty} \sum_{i=0}^{\infty}\left\langle v_{j}, v_{i}\right\rangle \\
& \leqslant \sum_{j=0}^{\infty} \sum_{i=0}^{\infty}\left(\chi^{(i-j) / 2}\left|v_{j}\right|\right)\left(\chi^{(j-i) / 2}\left|v_{i}\right|\right) \\
& \leqslant \sum_{j=0}^{\infty} \sum_{i=0}^{\infty} \frac{1}{2}\left(\chi^{i-j}\left|v_{j}\right|^{2}+\chi^{j-i}\left|v_{i}\right|^{2}\right) \\
& =\sum_{j=0}^{\infty} \sum_{i=0}^{\infty} \chi^{i-j}\left|v_{j}\right|^{2} \\
& =\sum_{j=0}^{\infty} \frac{\chi^{-j}}{1-\chi}\left|v_{j}\right|^{2} .
\end{aligned}
$$


We now bound the right-hand side of (4.1). Fix $\lambda^{2}<\chi<1$. Using Lemmas 4.4 and 4.5 and writing $\pi=\left(\pi_{1}, \pi_{2}, \ldots, \pi_{m}\right)$, we have

$$
\begin{aligned}
4 \sum_{i=0}^{2^{k}-1} \max _{\rho \in P_{\omega_{1} \cdots \omega_{k}}}\left|\sum_{j=k+1}^{\infty} \pi\left(\rho_{j}\right)\right|^{2} & \leqslant \frac{4}{1-\chi} \sum_{i=0}^{2^{k}-1} \max _{\rho \in P_{\omega_{1} \cdots \omega_{k}}} \sum_{j=k+1}^{\infty} \chi^{k+1-j}\left|\pi\left(\rho_{j}\right)\right|^{2} \\
& \leqslant \frac{4}{1-\chi} \sum_{n=1}^{m} \sum_{i=0}^{2^{k}-1} \max _{\rho \in P_{\omega_{1} \cdots \omega_{k}}} \sum_{j=k+1}^{\infty} \chi^{k+1-j} \pi_{n}\left(\rho_{j}\right)^{2} \\
& \leqslant \frac{4 m}{1-\chi} \sum_{j=k+1}^{\infty} \chi^{k+1-j} 2 \lambda^{2(j-1)} \\
& =\frac{8 m \chi^{k}}{(1-\chi)} \sum_{j=k+1}^{\infty}\left(\frac{\lambda^{2}}{\chi}\right)^{j-1} \\
& =\frac{8 m \lambda^{2 k}}{(1-\chi)\left(1-\lambda^{2} / \chi\right)}
\end{aligned}
$$

Setting

$$
C_{4}=\frac{8 m}{(1-\chi)\left(1-\lambda^{2} / \chi\right)},
$$

the proof of Proposition 4.3 is complete.

\section{REFERENCES}

[1] C. Beck. Upper and lower bounds on the Renyi dimensions and the uniformity of multifractals. Physica D 41 (1990), 67-78.

[2] A. Ben-Artzi, A. Eden, C. Foias and B. Nicolaenko. Hölder continuity for the inverse of Mañé's projection. J. Math. Anal. Appl. 178(1) (1993), 22-29.

[3] V. Chepyzhov and M. Vishik. Attractors for Equations of Mathematical Physics. American Mathematical Society, Providence, RI, 2002.

[4] A. Eden, C. Foias, B. Nicolaenko and R. Temam. Exponential Attractors for Dissipative Evolution Equations. Wiley, 1994.

[5] K. Falconer. Fractal Geometry: Mathematical Foundations and Applications. Wiley, New York, 1990.

[6] K. Falconer and J. D. Howroyd. Projection theorems for box and packing dimensions. Math. Proc. Cambridge Philos. Soc. 119(2) (1996), 287-295.

[7] C. Foias and E. Olson. Finite fractal dimension and Hölder-Lipschitz parametrization. Indiana Univ. Math. J. 45(3) (1996), 603-616.

[8] P. Friz and J. Robinson. Smooth attractors have zero 'Thickness'. J. Math. Anal. Appl. 240(1) (1999), $37-46$.

[9] R. J. Gardner. The Brunn-Minkowski Inequality. Bull. Amer. Math. Soc. 39 (2002), 355-405.

[10] P. Grassberger. Generalized dimension of strange attractors. Phys. Lett. 97A (1983), 227-231.

[11] H. G. Hentschel and I. Procaccia. The infinite number of generalized dimensions of fractals and strange attractors. Physica D 8 (1983), 435-444.

[12] B. Hunt and V. Kaloshin. How projections affect the dimension spectrum of fractal measures. Nonlinearity 10 (1997), 1031-1046.

[13] B. Hunt and V. Kaloshin. Regularity of embeddings of infinite-dimensional fractal sets into finitedimensional spaces. Nonlinearity 12 (1999), 1263-1275.

[14] B. Hunt, J. Kennedy, T. Y. Li and H. Nusse. SLYRB measures: natural invariant measures for chaotic systems. Physica D 170(1) (2002), 50-71. 
[15] B. Hunt, T. Sauer and J. Yorke. Prevalence: a translation-invariant 'almost every' on infinite-dimensional spaces. Bull. Amer. Math. Soc. 27 (1992), 217-238.

[16] B. Hunt, T. Sauer and J. Yorke. Prevalence: an addendum. Bulletin Amer. Math. Soc. 28(2) (1993), 306-307.

[17] M. Järvenpää. On the upper Minkowski dimension, the packing dimension, and orthogonal projections. Ann. Acad. Sci. Fenn. Ser. A1 Math. Dissertationes 99 (1994), 1-34.

[18] V. Yu. Kaloshin. Some prevalent properties of smooth dynamical systems. Proc. Steklov Inst. Math. 213(2) (1996), 115-140.

[19] R. Kaufman. On Hausdorff dimension of projections. Mathematika 15 (1968), 153-155.

[20] J. M. Marstrand. Some fundamental geometrical properties of plane sets of fractional dimension. Proc. London Math. Soc. 4 (1954), 257-302.

[21] P. Mattila. Hausdorff dimension, orthogonal projections and intersections with planes. Ann. Acad. Sci. Fennicae A 1 (1975), 227-244.

[22] P. Mattila. Geometry of Sets and Measures in Euclidean Spaces. Cambridge University Press, Cambridge, 1995.

[23] J. C. Oxtoby. Measure and Category. Springer, New York, 1971.

[24] A. Rényi. Probability Theory. North-Holland, Amsterdam, 1970.

[25] R. H. Riedi. An improved multifractal formalism and self-affine measures. J. Math. Anal. Appl. 189 (1995), 462-490.

[26] J. Robinson. Infinite-Dimensional Dynamical Systems. Cambridge University Press, 2001.

[27] T. Sauer and J. Yorke. Are the dimensions of a set and its image equal under typical smooth functions? Ergod. Th. \& Dynam. Sys. 17(4) (1997), 941-956.

[28] G. Sell and Y. You. Dynamics of Evolutionary Equations. Springer, 2002.

[29] R. Temam. Infinite-Dimensional Dynamical Systems in Mechanics and Physics. Springer, 1997.

[30] L.-S. Young. What are SRB measures, and which dynamical systems have them? Dedicated to David Ruelle and Yasha Sinai on the occasion of their 65th birthdays. J. Stat. Phys. 108(5-6) (2002), 733-754. 